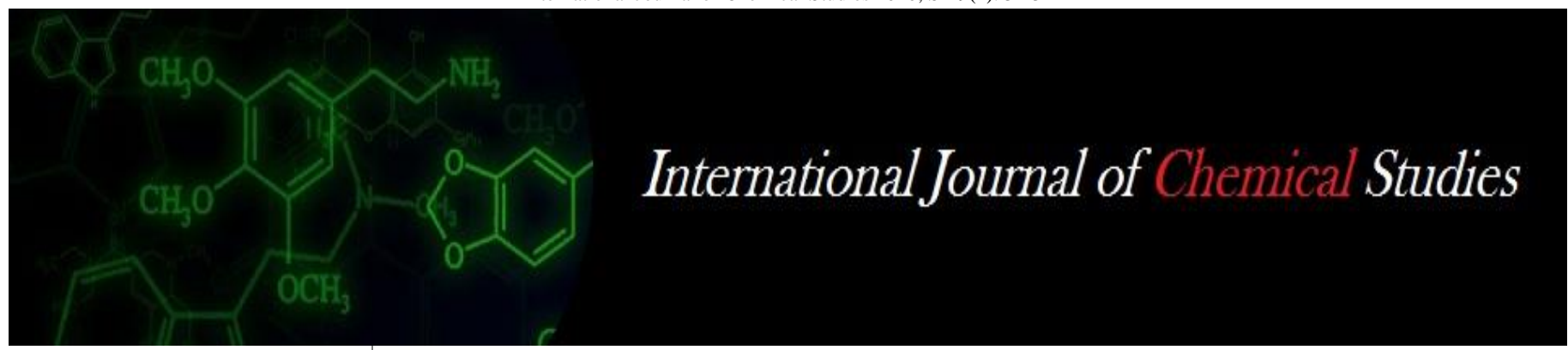

P-ISSN: 2349-8528

E-ISSN: 2321-4902

www.chemijournal.com

IJCS 2021; SP-9(1): 31-34

(C) 2021 IJCS

Received: 14-11-2020

Accepted: 16-12-2020

\section{Gurpreet Dhillon}

Guru Nanak Girls College,

Ludhiana, Punjab, India

\section{Sandeep Bains}

Department of Apparel and

Textile Science, Punjab

Agricultural University,

Ludhiana, Punjab, India

Sumeet Grewal

Department of Apparel and

Textile Science, Punjab

Agricultural University,

Ludhiana, Punjab, India

Devinder K Kocher

Department of Zoology, Punjab

Agricultural University,

Ludhiana, Punjab, India

\section{Mosquito repellent eucalyptus and rosemary essential oil finishes for cotton fabric}

\author{
Gurpreet Dhillon, Sandeep Bains, Sumeet Grewal and Devinder K Kocher
}

DOI: https://doi.org/10.22271/chemi.2021.v9.i1a.11378

\begin{abstract}
The present paper furnishes the mosquito repellency of microencapsulated textile finishes developed using two essential oils- eucalyptus (Eucalyptus globulus) and rosemary (Rosmarinus officinalis) for cotton fabric. The microencapsulated finishes were prepared taking 10 percent concentration of each essential oil as the effect of the application at this concentration on the physical properties of the fabric was less and in permissible limits. The prepared finishes were applied on the fabric through pad-dry-cure method. The effect of microencapsulated finishes on the selected textile properties such GSM, fabric thickness, tensile strength, bending length and wash fastness was studied. The results showed that GSM, fabric thickness and bending length slightly increased after the application of microencapsulated finishes, whereas the tensile strength somewhat decreased. A cage test was conducted for testing the efficacy of the developed microencapsulated finishes against Aedes, Anopheles and Culex mosquito species. It was found that the eucalyptus oil microencapsulated finish provided 100 percent protection from Aedes mosquitoes even after 15 washings, whereas the protection from Anopheles and Culex species was less which further got reduced after successive washings. On the other hand, rosemary essential oil showed lesser protection, between 40-49 percent for all three mosquito species. The efficacy of the finish also decreased after repeated washings. Hence, eucalyptus oil was found to be more effective as compared to rosemary essential oil in repelling the mosquitoes.
\end{abstract}

Keywords: Essential oil, microencapsulation, mosquito, repellency, textile finish

\section{Introduction}

Mosquitoes are medically most significant vectors that transmit parasites and pathogens, which continue to have an over whelming impact on the human beings. They are the main cause of spreading deadly diseases like malaria, dengue, filariasis and chikunguniya. There is no effective vaccine against malaria and dengue, the only way to avoid it, remains avoiding mosquito bites. Different volatile organic molecules have the potential to inhibit the scent orientation of insects in confined environmental conditions and therefore can be efficiently used as repellants against mosquito populations.

Repellency is one of the commonly used methods to prevent mosquitoes and other blood sucking pests from biting. Repellants are applied directly on the skin or to clothing and other fabrics such as bed-nets and anti-mosquito screens. The prolonged use of commercially developed mosquito coils, mats and vaporizers which contain synthetic pyrethroids like pyrethrin, allethrin, parallethrin and chemical such as N, N-diethyl-m-toluamide (DEET) may cause harmful effects and the mosquitoes develop resistance to these chemicals as well ${ }^{[1]}$. In the commercial mosquito vapourisers, the synthetic and chemical insecticides are dissolved in deodorized kerosene, which also causes side effects to the user. Due to some of the drawbacks of synthetic (chemical) insecticides, including lack of selectivity, environmental contamination, and the emergence and spread of vector resistance, development of natural products for vector control has always been the interest. Among the plant possessing pest control/repellent properties, around 300 species have been reported to contain bioactive materials showing some repellent activity against mosquitoes ${ }^{[2]}$.

In search of alternative and safe methods to protect from mosquito bites, products from essential oils are emerging as good mosquito repellants and they are effective, safe and also of low cost ${ }^{[3]}$. The use of the repellents such as lotions, coils and liquidators are limited in their use due to various reasons. This has necessitated the development of mosquito repellent fabrics.
Corresponding Author: Sumeet Grewal

Department of Apparel and

Textile Science, Punjab

Agricultural University,

Ludhiana, Punjab, India 
Protective textiles refer to those textile products which have a functionality of giving protection from something in some or the other sense. Mosquito repellent finished textiles are also a part of protective textiles which help in protection from species that are prone to cause damage. Mosquito repellent textiles is one of the revolutionary ways to advance the textile field by providing the much needed feature for driving away mosquitoes ${ }^{[4]}$. The present study was planned with an aim to test the mosquito repellency of developed microencapsulated textile finishes from two essential oils as the study of their repellent activity on textile material is limited and also not well documented.

\section{Materials and Methods \\ Materials}

The material used for the study include $100 \%$ pure cotton fabric, eucalyptus and rosemary essential oils as core material and gum acacia as wall material, sodium sulphate and citric acid were used as cross linking agents. Cotton fabric was scoured to remove impurities. To confirm that the fabric procured for the study was pure cotton, its microscopic, burning and chemical tests were conducted. Fabric thread count was determined by a pick glass. The thread count of the substrate was 30x 35 ends and picks per inch. The GSM (grams per square meter) of the cotton fabric was found to be $152 \mathrm{~g} / \mathrm{m}^{2}$. The essential oils were selected after studying the properties and factors such as mosquito repellency, their nonallergenic behavior to humans and cost effectiveness ${ }^{[5]}$.

\section{Optimization of concentration of essential oils}

To optimize the concentration of eucalyptus and rosemary essential oils, the solution was applied on the fabric using pad-dry-cure method. Three concentrations of essential oil i.e. 10,30 and $50 \%$ were used for optimization where ph, time and temperature will be kept constant. The effect of application of the essential oil at different concentrations was studied on the selected textile properties such as tensile strength, bending length and whiteness index. Computerized tensile strength tester was used for measuring strength, stiffness tester for measuring bending length and color flex spectrophotometer for measuring the whiteness index. The concentration at which the physical properties were least affected, was selected for preparing microencapsulated essential oil finishes.

\section{Development and application of microcapsules}

Essential oils were used as the core of the microcapsules while gum acacia as the shell. Gum acacia was allowed to swell for a ten minutes in hot water. Stirring was done with a magnetic stirrer and the temperature was maintained at about $40^{\circ} \mathrm{C}$. To this suspension core material was added under regular stirring condition. Sodium sulphate and citric acid were used as auxiliaries for making microcapsules. The prepared microcapsules were freeze dried for $8 \mathrm{hrs}$. Microcapsules thus obtained were applied on the fabric through pad-dry-cure method.

\section{Effect of microencapsulated finishes on selected fabric properties}

The effect of microencapsulated finishes on selected textile properties such as GSM, thickness, tensile strength, bending length and whiteness index were studied ${ }^{[6]}$. The efficacy of the developed finish was studied by washing the finished fabrics with a pre-determined number (three) of washing cycles in launder-o-meter using standard test recommended by AATCC: 43-1995. One wash cycle is equivalent to five simple home launderings. After each wash cycle, mosquito repellency was tested using Cage test.

Cage test for repellency assessment of the finished fabrics For the conduct of this experiment, two types of gloves were stitched and were labeled as treated gloves (stitched from microencapsulated fabric) and the untreated/control gloves (stitched from normal/untreated textile). The experiment was designed to observe the mosquito landing on the oil treated and untreated gloves and for this purpose 5-7 days old 30 adult mosquitoes of different types i.e. Aedes, Anopheles and Culex which were kept in separate cages. Five subjects (volunteers) were selected and provided with gloves treated with microcapsules of eucalyptus and rosemary oil as well as untreated gloves. The volunteers introduced their forearm covered with microencapsulated fabric glove and untreated fabric glove inside the cage (having one type of adult mosquitoes) for $3 \mathrm{~min}$ without touching the sleeves in order to prevent a treatment from being wiped off and contaminating the closure. The sleeves were wrapped up behind the elbow to avoid the escape of mosquitoes. During these $3 \mathrm{~min}$ stay, the number of visits/landings done by the mosquitoes to the glove were counted. This observation was repeated for three times with an interval of 5 min every time. The first testing (before washing) of gloves was followed by the testing done after 5 washings, then 10 times washing and lastly after 15 times washings with non scented soap by following the above said method. At least 2 mosquitoes should visit/land in the trial otherwise the trial was not taken into consideration and was performed again. The time between applications of the treated materials recorded as the protection time. The percentage of repellency or mosquito protection was calculated by using the regularly utilized formula given below ${ }^{[7,8]}$.

Mosquito protection $(\%)=\frac{(\mathrm{U}-\mathrm{T})}{\mathrm{U} \times 100}$

Where $U$ corresponds to the number of mosquitoes visited/landed on untreated gloves and $\mathrm{T}$ represents the number of mosquitoes visited/landed on treated gloves.

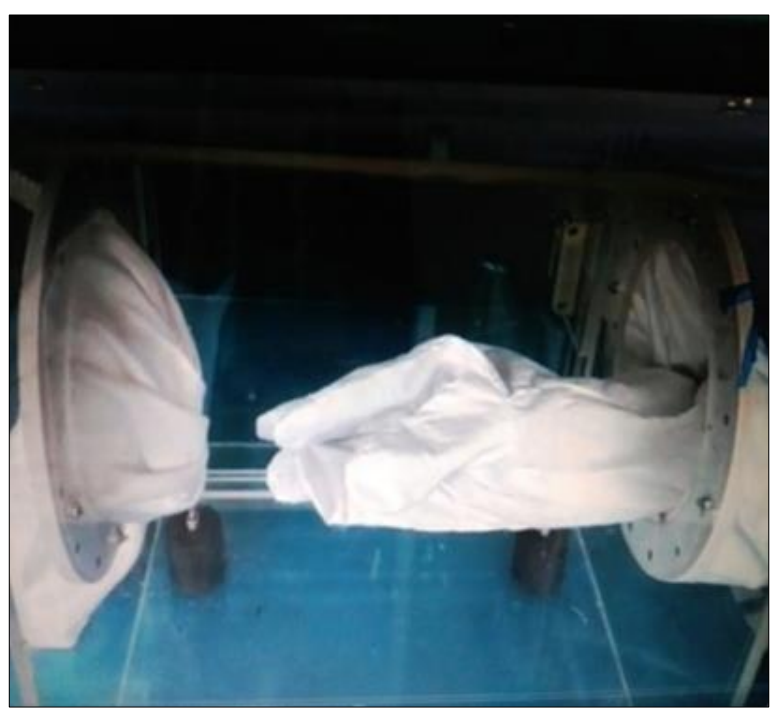

Fig 1: Oil treated glove 


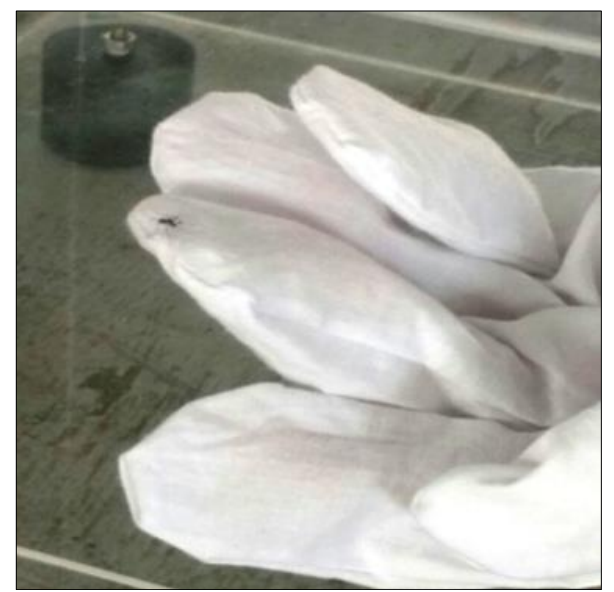

Fig 2: Untreated glove with landed mosquito
Comparative analysis of mosquito protection (\%) from Aedes, Anopheles and Culex species by using microencapsulated fabric was calculated with the help of SPSS statistical software version 16 using ANOVA (Duncan multiple range test) at 5 percent level of significance.

\section{Results and Discussion \\ Optimization of concentration of eucalyptus and rosemary essential oils}

Eucalyptus and rosemary essential oils were applied on the fabric using pad-dry-cure method in three concentrations 10 , 30 and $50 \%$ where ph, time and temperature were kept constant ${ }^{[9]}$. The effect of application of the essential oils at different concentrations was studied on the selected textile properties such as tensile strength, bending length and whiteness index.

Table 1: Effect of concentration of eucalyptus and rosemary essential oils on selected fabric properties

\begin{tabular}{|c|c|c|c|c|c|c|c|c|}
\hline \multirow{2}{*}{ Tensile strength $\left(\mathbf{N} / \mathbf{m}^{\mathbf{2}}\right)$} & \multicolumn{3}{|c|}{ Concentration of eucalyptus oil } & \multicolumn{3}{|c|}{ Concentration of rosemary oil } \\
\cline { 2 - 8 } & Control & $\mathbf{1 0 \%}$ & $\mathbf{3 0 \%}$ & $\mathbf{5 0 \%}$ & $\mathbf{1 0} \%$ & $\mathbf{3 0 \%}$ & $\mathbf{5 0 \%}$ \\
\hline Warp & 26.43 & 26.35 & 26.05 & 25.83 & 26.33 & 26.08 & 25.06 \\
\hline Weft & 23.14 & 22.78 & 22.19 & 21.11 & 23.15 & 22.15 & 21.19 \\
\hline \multicolumn{7}{|c|}{ Bending length (cm) } \\
\hline Warp & 2.13 & 2.29 & 2.44 & 2.51 & 2.45 & 2.89 & 2.89 \\
\hline Weft & 2.08 & 2.26 & 2.31 & 2.53 & 2.56 & 2.62 & 2.62 \\
\hline \multicolumn{7}{|c|}{ Whiteness Index } \\
\hline L-values & 91.00 & 90.15 & 88.15 & 87.07 & 91.00 & 89.19 & 88.15 \\
\hline a-values & 3.55 & 3.15 & 2.37 & 2.32 & 3.52 & 2.85 & 2.25 \\
\hline b-values & 24.00 & 22.5 & 14.22 & 10.75 & 23.5 & 12.75 & 11.75 \\
\hline
\end{tabular}

As evident from Table 1, the tensile strength of the fabrics decreased after application of essential oil finish but the samples treated with $10 \%$ concentration of eucalyptus and rosemary essential oil showed minimum decrease in the warp and weft direction. At $30 \%$ concentration the tensile strength again decreased slightly, whereas at $50 \%$ concentration it further decreased. Hence, with the increase in the concentration of the oils, the tensile strength of the fabric decreased. On the other hand, the bending length of the treated fabrics increased with the increase in the concentration of each essential oil, which indicates that the fabric became stiff as a result of higher concentration of oil. The Whiteness Index parameters depicted by $\mathrm{L}$ values show that the decrease in the values with increase in the concentration of oils in the finish resulted in the fabrics becoming darker. Whereas, the decreasing $a$ and $b$ values depict that the treated fabrics became less red and less yellow with the increase in the concentration of oil. Hence, 10 percent concentration was selected for microencapsulation as it had minimum effect on the selected textile properties.

\section{Effect of microencapsulated finishes on selected fabric properties}

The physical properties have greater influence and play an important role in determining the quality of the fabric. The physical properties were assessed to determine the appearance, performance and serviceability of the fabric. The test samples were assessed for fabric weight (GSM), thickness, tensile strength, bending length using Standard Test Methods and the results are furnished in following tables.
Table 2: Effect of microencapsulated finishes on GSM and thickness of the fabric

\begin{tabular}{|c|c|c|c|c|}
\hline Fabric Samples & \multicolumn{2}{|c|}{ GSM(g/m $\left.\mathbf{m}^{\mathbf{2}}\right)$} & \multicolumn{2}{c|}{ Fabric thickness (mm) } \\
\hline & Eucalyptus & Rosemary & Eucalyptus & Rosemary \\
\hline Untreated & 152.25 & 152.25 & 0.80 & 0.80 \\
\hline Microencapsulated & 158.98 & 158.95 & 0.84 & 0.85 \\
\hline
\end{tabular}

The weight (GSM) is defined as the weight of specimen in one square meter of length. From Table 2, it is clear that with the application of microencapsulated finish, the GSM and thickness of the fabric increased. Whenever, any finish is applied to the fabric it is bound to increase its weight and also adds to its thickness.

Table 3: Effect of microencapsulated finishes on tensile strength of the fabric

\begin{tabular}{|c|c|c|c|c|}
\hline \multirow[t]{2}{*}{ Fabric Samples } & \multicolumn{2}{|c|}{$\begin{array}{c}\text { Tensile strength } \\
\text { (warp ) N/m }\end{array}$} & \multicolumn{2}{|c|}{$\begin{array}{l}\text { Tensile strength } \\
\text { (weft) } \mathrm{N} / \mathrm{m}^{2}\end{array}$} \\
\hline & Eucalyptus & Rosemary & Eucalyptus & Rosemary \\
\hline Untreated & 26.43 & 26.43 & 23.14 & 23.14 \\
\hline Microencapsulation & 25.53 & 24.96 & 21.26 & 21.30 \\
\hline
\end{tabular}

Table 3 depicts the effect of microencapsulated finish on the tensile strength of the fabric. It decreased in warp direction after application of both the eucalyptus and rosemary microcapsules. The tensile strength of the fabric also decreased in weft direction. But the decrease was in permissible limit, therefore, the strength of fabric was not effected much. 
Table 4: Effect of microencapsulated finishes on bending length of the fabric

\begin{tabular}{|c|c|c|c|c|}
\hline \multirow[t]{2}{*}{ Application method } & \multicolumn{2}{|c|}{$\begin{array}{c}\text { Bending length } \\
\text { (warp) } \mathrm{cm}\end{array}$} & \multicolumn{2}{|c|}{\begin{tabular}{|c|}
$\begin{array}{c}\text { Bending length } \\
\text { (weft) } \mathrm{cm}\end{array}$ \\
\end{tabular}} \\
\hline & Eucalyptus & Rosemary & Eucalyptus & Rosemary \\
\hline Untreated & 2.93 & 2.93 & 2.88 & 2.88 \\
\hline Microencapsulation & 2.98 & 3.04 & 2.93 & 2.98 \\
\hline
\end{tabular}

Bending length is the length of the fabric that will bend under its own weight to a definite extent. It is a measure of the stiffness that determines draping quality. Table 4 illustrates that after microencapsulation treatment fabric showed increase in the bending length. Therefore, it can be concluded that bending length increased when finishing treatment was applied to the fabric and it became stiff.

\section{Results of the Cage test for testing the mosquito repellency of the microencapsulated finished fabrics}

Data furnished in table 5 reveals that the gloves prepared from eucalyptus and rosemary oil treated fabrics showed 100 percent protection from Aedes mosquitoes before washing. Eucalyptus oil treated gloves provided efficient repellency potential against Aedes mosquito as 100 percent protection was observed even after 15 washings. On the other hand rosemary treated fabrics showed 100 percent protection till 5 washings, later on this protection was reduced. The essential oils gave good repellency against Aedes mosquito and the microencapsulated oil treated fabric showed higher repellency than the direct oil treated fabric. This indicates that microencapsulated oil release from the capsule is due to controlled release nature of cross linked natural polymers ${ }^{[9]}$.

Table 5: Comparative analysis of protection (\%) provided by the fabrics treated with essential oil finishes against Aedes, Anopheles and Culex mosquito

\begin{tabular}{|c|c|c|c|c|}
\hline $\begin{array}{c}\text { Oil treated } \\
\text { textile }\end{array}$ & $\begin{array}{c}\text { Before } \\
\text { washing }\end{array}$ & $\begin{array}{c}\text { After 5 } \\
\text { washings }\end{array}$ & $\begin{array}{c}\text { After 10 } \\
\text { washings }\end{array}$ & $\begin{array}{c}\text { After 15 } \\
\text { washings }\end{array}$ \\
\hline \multicolumn{5}{|c|}{ Aedes Mosquito } \\
\hline Eucalyptus & $100.00 \pm 0.00^{\mathrm{a}}$ & $100.00 \pm 0.00^{\mathrm{b}}$ & $100.00 \pm 0.00^{\mathrm{c}}$ & $100.00 \pm 0.00^{\mathrm{c}}$ \\
\hline Rosemary & $100.00 \pm 0.00^{\mathrm{a}}$ & $100.00 \pm 0.00^{\mathrm{b}}$ & $58.64 \pm 2.86^{\mathrm{a}}$ & $54.45 \pm 2.87^{\mathrm{a}}$ \\
\hline \multicolumn{5}{|c|}{ Anopheles Mosquito } \\
\hline Eucalyptus & $62.03 \pm 3.88^{\mathrm{b}}$ & $37.57 \pm 3.31^{\mathrm{a}}$ & $45.23 \pm 4.48^{\mathrm{c}}$ & $34.76 \pm 4.98^{\mathrm{b}}$ \\
\hline Rosemary & $40.36 \pm 3.73^{\mathrm{a}}$ & $40.29 \pm 5.11^{\mathrm{b}}$ & $33.33 \pm 5.74^{\mathrm{a}}$ & $30.78 \pm 3.88^{\mathrm{a}}$ \\
\hline \multicolumn{5}{|c|}{ Culex Mosquito } \\
\hline Eucalyptus & $64.65 \pm 4.02^{\mathrm{b}}$ & $54.58 \pm 6.30^{\mathrm{b}}$ & $51.08 \pm 5.24^{\mathrm{c}}$ & $52.35 \pm 4.24^{\mathrm{c}}$ \\
\hline Rosemary & $43.23 \pm 3.30^{\mathrm{a}}$ & $46.04 \pm 3.88^{\mathrm{a}}$ & $39.21 \pm 4.15^{\mathrm{a}}$ & $34.65 \pm 7.48^{\mathrm{a}}$ \\
\hline - Number of mosquitoes taken during each trial = 30 \\
- Number of subjects $=5$ \\
- Time taken for each observation $=3$ minutes \\
- Number of replicates $=3$ \\
- All Values are Mean \pm S.D \\
- Figures followed with different superscripts indicate significant \\
difference $(p<0.05)$ by using Duncan multiple range test
\end{tabular}

It is also evident from the table that none of the treated gloves offered 100 percent protection from Anopheles mosquitoes even prior to washing. Maximum protection from Anopheles mosquitoes was found to be 62.03 percent with eucalyptus oil treated gloves and 40.36 percent with rosemary oil treated gloves before washing which was further reduced after 5, 10 and 15 washings. Eucalyptus essential oil has a very good repellent effectiveness $(97.15 \%)$ against Anopheles mosquitoes. Hence, it could be recommended as a safe and suitable substitute of chemical repellent ${ }^{[10]}$. Similarly, none of the oil treated gloves were found to show 100 percent protection from Culex mosquitoes even before washing.
Maximum protection from Culex mosquitoes was found to be 64.65 percent with eucalyptus oil treated gloves and 43.23 percent with rosemary oil treated gloves before washing which further reduced after 5, 10 and 15 washings. Essential oils C Citratus and C Nardus also gives good repellency i.e. 92 percent and 82 percent respectively against Culex mosquitoes ${ }^{[11]}$.

\section{Conclusion}

The mosquito repellency and wash fastness results of eucalyptus shows a promising future for commercial development of the mosquito repellant cotton fabric which can be utilized for production of many end products like wrist bands, socks, pillow covers, bed sheets and apparel items. Currently, a variety of synthetic repellents mainly containing $\mathrm{N}, \mathrm{N}$-diethyl-m-toluamide (DEET) as an active ingredient are available in the market. These repellents cause side effects like breathing problems, eye irritation, headache, cough, asthma etc. Whereas essential oils provide a very good option for mosquito repellency, as they are safe for human use and are biodegradable. Hence, essential oils especially eucalyptus which show very good repellency against various mosquito species offer an eco-friendly, non-toxic alternative finish on fabrics to safeguard humans from vector borne diseases.

\section{References}

1. Maguranyi SK, Webb CE, Mansfield S, Russel RC. Are commercially available essential oils from Australian native plants repellent to mosquitoes? Journal of American Mosquito Control Association 2009;25:292-300.

2. Gunasekaran, Vijayakumar T, Kalyanasundaram M. Larvicidal \& emergence inhibitory activities of Neem Azal T/S1.2 percent EC against vectors of malaria, filariasis \& dengue. Indian Journal of Medical Research 2008;130:138-45.

3. Mittal PK, Subbarao SK. Prospect of using herbal product in the control of mosquito vectors. ICMR Bulletin 2003;33:0377-4910.

4. Prabha R, Vasugi RN. Filarial repellent finish using medicinal plants. Indian Journal of Science 2012;1:74-76.

5. Maia M, Moore S. Plant based insect repellents: A review of their efficacy, development and testing. Malaria Journal 2011;35:128-29.

6. Sandhu G. Development of micro-encapsulated mosquito repellent textile finish using essential oils. Doctorate Thesis. Punjab Agricultural University, Ludhiana 2016.

7. WHO. Guidelines for the efficacy testing of mosquito repellents for human skin 2009.

8. Lupi E, Hatz C, Schlagenhauf P. The efficacy of repellents against Aedes, Anopheles, Culex and Ixodes spp. - A literature review. Travel Medical Infectious Diseases 2013;11(6):374-411.

9. Vigneshkumar M, Mohamed K, Vijayakumar V. Potential and controlled repellent activity of microencapsulated citronella oil treated textile cotton fabrics against Aedes aegypti ISSN: 2277-2197. Hitek Journal of Bioscience and Bioengineering 2012;1(1):124-26.

10. Gillij YG, Gleiser RM, Zygadlo JA. Mosquito repellent activity of essential oils of aromatic plants growing in Argentina. Bioresearch Technology 2008;99:2507-2515.

11. Siriporn P, Soonwera M. Comparative mosquito repellency of essential oils against Aedes aegypti (Linn.), Anopheles dirus (Peyton and Harrison) and Culex quinquefasciatus (say). Asian Pacific Journal of Tropical Biomedicine 2011;4:113-118. 